\title{
PHEN: Parkinson Helper Emergency Notification System using Bayesian Belief Network
}

\author{
Hamid Mcheick ${ }^{1}$, Malak Khreiss ${ }^{2}$, Hala Sweidan², Iyad Zaarour ${ }^{2}$ \\ ${ }^{1}$ Computer Science Department, University of Quebec At Chicoutimi, \\ 555, Boulevard de l'Université, Chicoutimi, Québec, Canada, G7H-2B1 \\ Hamid Mcheick@uqac.ca \\ ${ }^{2}$ Faculty of Sciences (I), Lebanese University, Hadath-Beirut, Beirut, Lebanon \\ malakkhreis@outlook.com, hala-sweidan@live.com, i.zaarour@ul.edu.lb
}

\begin{abstract}
Context-aware systems are used to aid users in their daily lives. In the recent years, researchers are exploring how context aware systems can benefit humanity through assist patients, specifically those who suffer incurable diseases, to cope with their illness. In this paper, we direct our work to help people who suffer from Parkinson disease. We propose PHEN, Parkinson Helper Engine Network System, a context-aware system that aims to support Parkinson disease patients on $\mathrm{m}$ any levels. We use ontology is for context representation and modeling. Then the ontology based context model is used to learn with Bayesian Belief network (BBN) which is beneficial in handling the uncertainty aspect of context-aware systems.
\end{abstract}

Keywords: Context-aware applications, Parkinson Disease, Health Care system, Bayesian Belief Network, Ubiquitous Computing, OWL, Telemedicine.

\section{Introduction}

Pervasive computing, also known as ubiquitous computing, have made a dramatical evolution in our century. Its main purpose is to create and correlate a strong environment between computing and communication components in order to adapt to the user's context. Context awareness is regarded as the enabling technology for pervasive computing systems.

However, one of the main problems of context aware system is uncertainty of context, which is unavoidable due to the nature of sensed data which could be imperfect, incomplete, wrong or ambiguous. Uncertainty is a situation of inadequate information [6] which can be of three sorts: inexactness, unreliability, and border with ignorance. As itself has many forms and dimensions and may include concepts such as fuzziness or vagueness, disagreement and conflict, imprecision and non-specificity [10]. Many context modeling and retrieval architectures tend to over-simplify uncertainty by assuming a perfect knowledge in combination with perfect inference [1]. Many techniques could be used to handle the uncertainty aspect of context aware 
systems, one of the most used techniques is the $\mathrm{BBN}$, which have been also used in fault detection and diagnosis of dynamic systems [11].

In this paper, we propose PHEN (Parkinson's Helper Engine using Network), a Bayesian based approach that aims to aid the Parkinson patients in their daily lives. Our system is designed to present relevant services for their users according to their needs. Specifically, during emergency situations.

This paper is organized as follows. Section II described briefly the related work, section III discuss our contribution. Section IV illustrates the results and interpretation. Finally section V summarizes the paper and gives directions for future work.

\section{Related Works}

BBN is widely used in context aware systems. In [7] the authors proposed SOCAM (Service-Oriented Context-Aware Middleware), to reason about uncertainty by providing supports to construct a BBN. The work in [2] shows the different benefits of using BBN in context-aware systems. Moreover, BBN was found to support fault detection in context ubiquitous systems [1]. Ontology, was found to be the perfect match for BBN. Examples of merging ontology with BBN in context-aware systems is the work proposed [15] and [16] where the authors used ontologies and BBN to propose a unified context model to support representation and reasoning about uncertain context using both relational schema and probabilistic models.

Assisting PD (Parkinson Disease) patients have also had a noticeable care by the research community. In [4] the authors developed a wearable assistant for PD patients with the FOG (freezing of gait) symptom. This wearable system uses onbody acceleration sensors to measure the patients' movements. It automatically detects FOG by analyzing frequency components inherent in these movements. Also, [12] presented GaitAssist, a system which is also designed for FOG detection and support in unsupervised areas. It uses Bluetooth to stream data for processing and detecting FOG, after that an audio notification is sent to the patient.

It is also noteworthy that in a previous work [9] we proposed CARE (ContextAware Reliable Engine) system, an AHP (Analytic Hierarchy Process) based contextaware system for health monitoring to support patients who could be subjected to serious emergencies.

\section{Motivational Scenario}

Sara is a 65 years old woman who lives with her grandson Peter. Recently, the symptoms of Parkinson disease started to appear on Sara.

Parkinson disease is a common neurological disorder caused by the progressive loss of dopaminergic and other subcortical neurons [3]. The loss of dopamine results in abnormal nerve-firing patterns within the brain that cause impaired movement [8]. The two most important problems the Parkinson disease (PD) patient may face are: 
Freezing of gait (FoG) and dysphagia. Freezing of gait is the state where the patients with Parkinson disease suffers from short periods during which they cannot continue walking and is associated to a fall risk and can be caused by a lot of triggers such as crowded places, turning corners, turning around in a circle. While the difficulty of swallowing which is called "dysphagia", it occurs while eating and can be fatal for patients with Parkinson disease. Sara was suffering from both symptoms.

To take care of Sara Peter bought PHEN, especially after hearing that PHEN can notify him when Sara has an emergency if she was alone at home. On a morning while Sara was walking at the backyard, wearing her wearable assistance, she started to feel something unusual happening to her legs. The PHEN system, using various sensors, directly detects FOG event by analyzing the frequency components of Sara's movements and sends an emergency notification to Peter and other neighbors that Peter entered their numbers to the system.

\section{Context Modeling}

\subsection{System Architecture}

PHEN system has four main functionalities:

- Detecting dysphagia

- Video tutorials for treatment

- Online physician consultant

- Health services

While eating, if the patient starts to cough, the PHEN server is able to detect dysphagia by analyzing the radio-frequency of the voice of the patient, and then it decide if it is a normal coughing or it is caused by the difficulty of swallowing. So the PHEN system sends a notification to the patient to give him some instructions to follow (e.g. Sit upright for at least 15 minutes after eating, drink frequent sips of water or suck on ice chips, reduce sugar intake, as sugar increases saliva, Take smaller bites of food, chew food thoroughly and eat slowly, take small sips of water or beverage when eating, drink tea with lemon or carbonated beverages to help thin phlegm, Sleep with head raised up to prevent choking). If the response is not achieved in few seconds, the system proposes that the patient is not able to react normally, so directly the system gives an emergency call to one of the patient's relatives.

In addition, PHEN system contains a set of video tutorials which consist of exercises to maintain strength and flexibility and treatments for difficulty swallowing (e.g. Take extra-small bites of food, chew thoroughly, and swallow carefully, take a breath before you start to speak, and pause between every few

words or even between each word). Besides, the patient who has the PHEN application can consult his physician online before starting with exercises or following a healthy diet. Moreover, PHEN system contains healthy services to check the blood pressure, amount of glucose, and heartbeats. Also, a service for defining the food's contents percentages. 
Figure-1 depicts the system architecture and its components. It is divided into three fundamental modules: Patient domain, PHEN application and PHEN server. This architecture is based on our exiting work $[18,19]$.

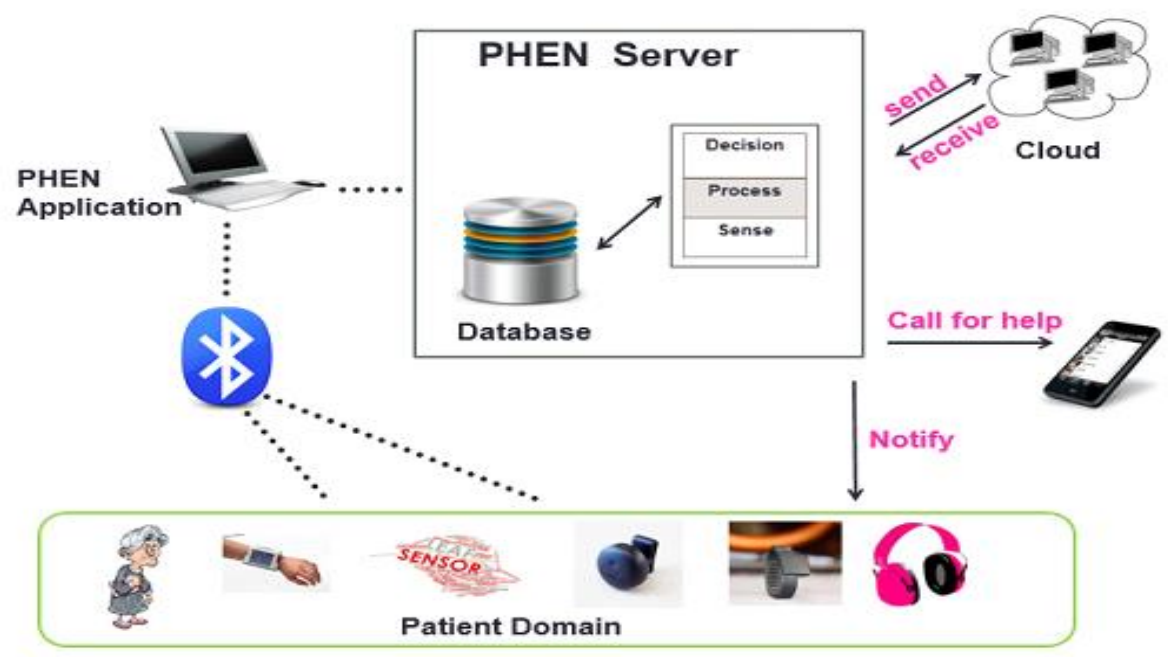

Fig. 1. PHEN system architecture.

The PHEN system is made up of three components: The patient domain, PHEN application, and PHEN server.

Patient domain: The patient domain consists of a patient wearing a FOG detection and feedback device, wearable computer, earphones and three sensors. The first sensor is attached to the shank (just above the ankle), the second to the thigh (just above the Knee) using elasticized strap and Velcro, and the third sensor to the belt where also a wearable computer is attached to. On the other hand, the patient wears earphones to receive notifications.

PHEN application: The PHEN application is an application that listens for incoming connections via Bluetooth from the wearable devices and the wearable computer. Once connected, the application starts to track the patient situation and sends data to the PHEN server directly.

PHEN server: The PHEN server has three layers. The first layer is responsible for retrieving the sensed data, it sends it to the upper layer to process and then directed to the last layer to take the decision. At each level, the database is updated (send and receive data). Once the system took the decision, it stores the appropriate data in the cloud and at the same time it either notify the PD patient by the decision taken, or call someone for help in case something urgent happened and the patient can't behave by himself. 


\subsection{Ontology}

Ontology is a formal, explicit description of concepts in a particular domain of discourse. It provides a vocabulary for representing domain knowledge and for describing specific situations in a domain [13]. In our work we use an ontology for modeling the profile of the user. The classes used in our ontology are: patient, location, state, situation, activity, wearable assistant, alarm and sensor.

\subsection{Probabilistic Model}

A probabilistic model is used as an extension to the ontology based model to represent uncertain context features. The probabilistic model has the form of Prob(Predicate(subject, value)) in which the probability measurement takes a value between 0 and 1 [7]. For example Prob(Activity(Patient,Eating)=0.96 means that the probability that the patient is eating is equal to $96 \%$.

Figure 2 depicts the context ontology for relational and probabilistic Knowledge, it describes the relation chains and the conditional probability dependency.

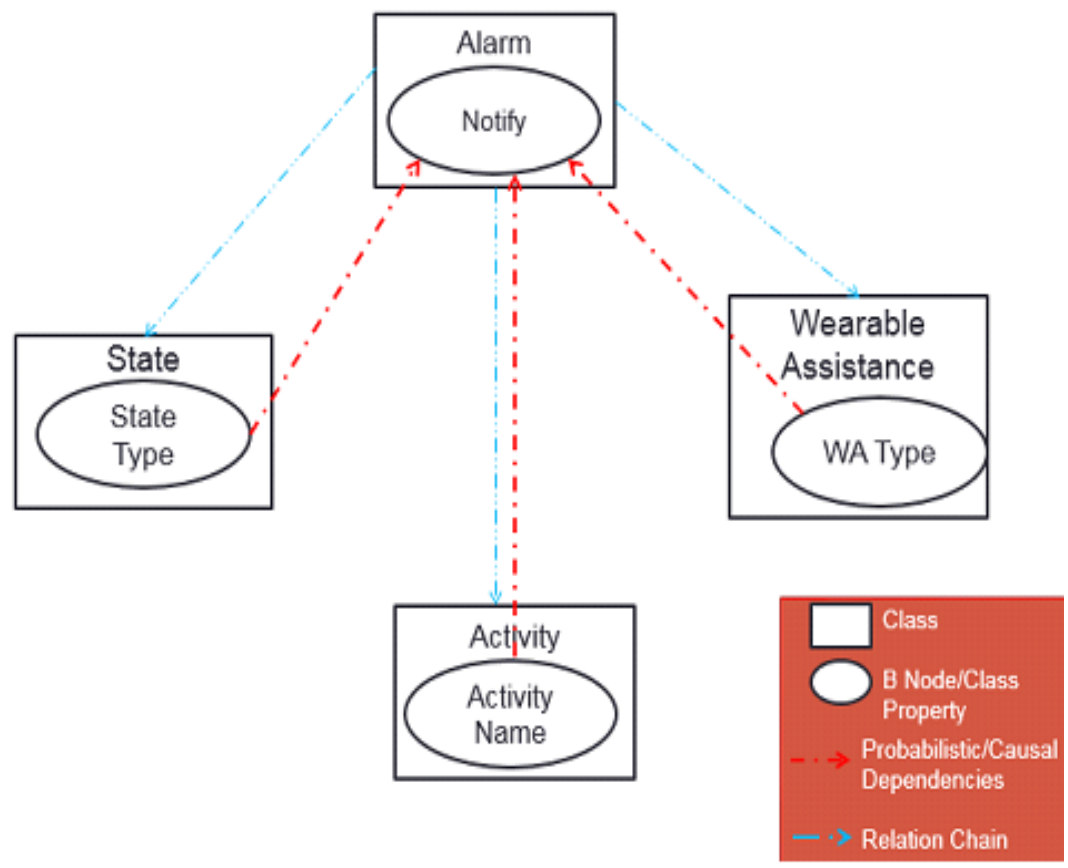

Fig. 2. PHEN Probabilistic Model. 


\section{Bayesian Belief Network Modeling}

The Bayesian belief network applied is a graphical, probabilistic model that represents cause and effect relationships [14]. It reflects the states of some part of a world that is being modeled and it describes how those states are related by probabilities.

The task of building the structure and assigning the probability distributions of a Bayesian Network is complex and knowledge-intensive [5]. During BBN construction nodes (variables of interest) and the relationships between these nodes must be identified. The transition from ontology to $\mathrm{BBN}$ is based on the approach presented in [15] where the authors developed a unified context ontology based on a given context model to which capture both structural and relational knowledge as well as the probabilistic knowledge of a domain. Then they derived in an autonomous and adaptive fashion which reflects most truthfully about the current state of the domain. Their BBN is constructed based on the OWL added concepts.

Fig. 3 depicts the updated context ontology after inference.

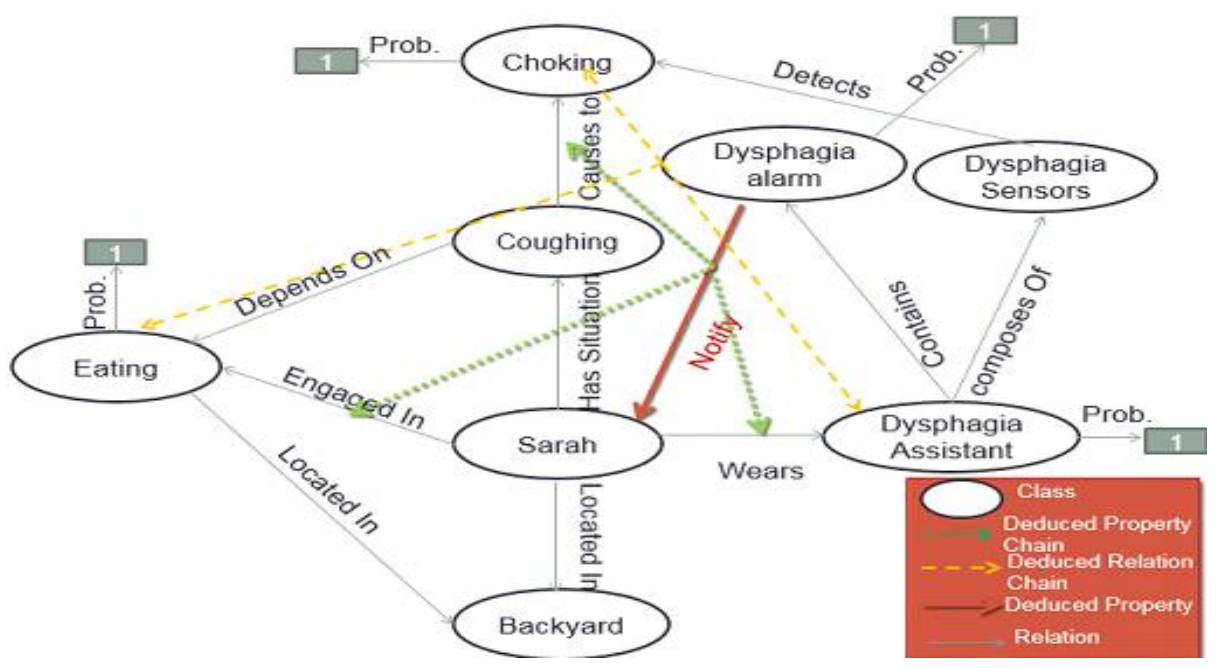

Fig. 3. Updated context ontology after inference.

We take into consideration the case where Sarah (the PD patient described in the earlier scenario) starts to face difficulty of swallowing while eating. After inference the probabilities of the activity Eating and the wearing assistance dysphagia that is responsible for detecting the choking cases, and that the state of the patient is choking are equal to 1 . So we can deduce a property to notify the patient or someone beside him that the patient is choking and that may lead to death. Based on the probabilistic model, and our domain specific ontology, we generate our Bayesian belief network as shown in figure. We conclude that the most three states that affect the alarm's notification are: the activity engaged in, the wearable assistant used, and the state of the patient noticed. Below is a simple Bayes net that illustrates these concepts. In this simple world, let us say the activity can have two binary states: eating or not, also that 
the wearable assistant can be dysphagia assist or no assist, furthermore the state of the patient can be choking or not and that the alarm notification can be yes or no.

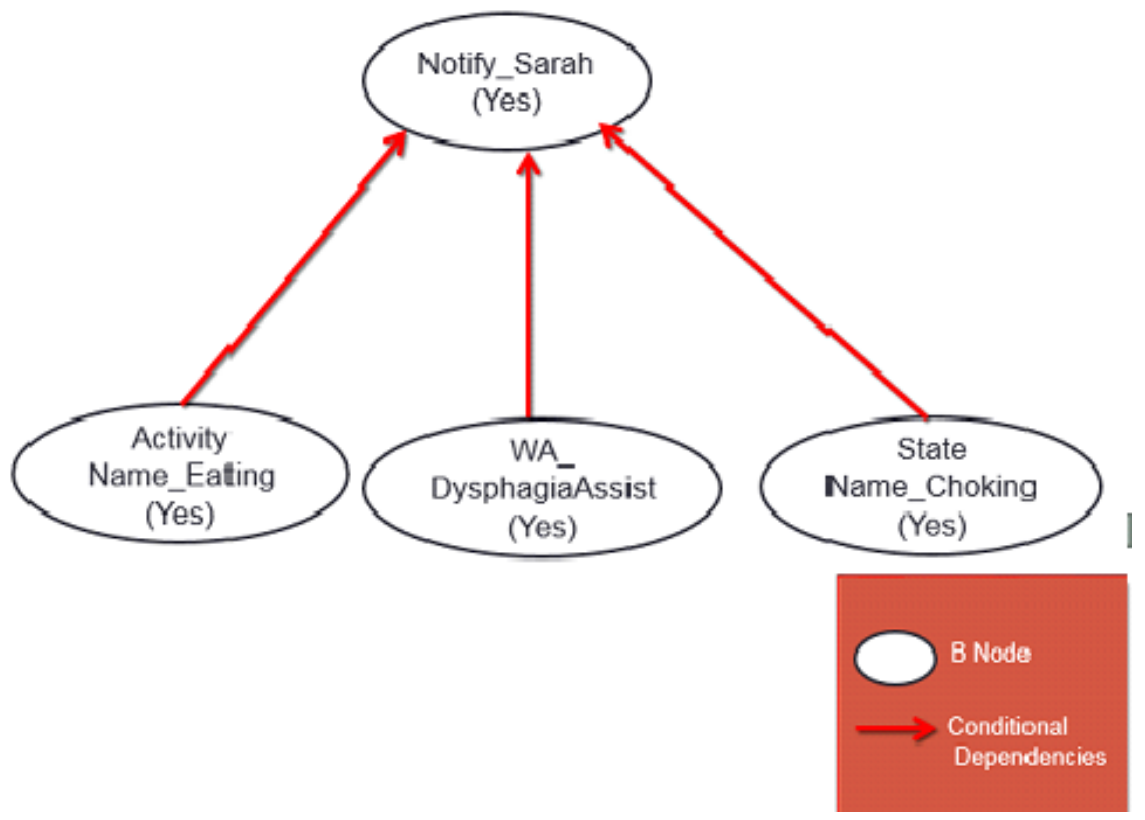

Fig. 4. PHEN Bayesian Belief Network.

Our BBN is constructed based on the OWL concepts. Where the nodes and the conditional probabilistic dependency depends on the individuals, deduced relation chain, deduced property chain, and deduced property. For example: To notify (deduced property), the conditional probabilistic dependency derived from the deduced property chain (ActivityName_Choking(Yes), WearableAssistant_DysphagiaAssist(Yes), and StateName_Choking(Y $\bar{Y} e s)$ ).

\section{Results and Discussion}

Netica [17] is used to implement the BBN. It is is a powerful, easy-to-use, complete program for working with belief networks and influence diagrams. The PHEN modeling process includes two stages. The first stage is building the model by determining the prior probabilities depending on the expert's knowledge. The second is interviewing experts to determine the posterior probabilities. Both stages will be discussed further in the upcoming sections.

\subsection{BBN Learning}

The BBN learning problem can be stated informally as follows: 
1. Health services Given training data and prior information.

2. Estimate the BBN structure (graph topology).

3. Estimate the BBN parameters.

Training Data: Guided and supervised by an expert, our training data is gathered. The primary objective in the interview process with the expert is to determine from resource professionals, a cause and effect of the patient-related activities and test our approach by real examples (data) in our daily life and translate our scenario in a real action, especially the results of the inference part provided by BBN.

Table 1. Prior Probabilities.

\begin{tabular}{|c|c|c|}
\hline & & Where: \\
\hline $\mathrm{P}(\mathrm{E} \mathrm{a}=\mathrm{T})$ & 0.88 & $P($ Ea $)=P($ Activity_Eating $)$ \\
\hline $\mathrm{P}(\mathrm{Ea}=\mathrm{F})$ & 0.12 & \\
\hline$P($ Dys $=T)$ & 0.9 & $P($ Dys $)=P($ WA_DysphagiaAssist) \\
\hline$P($ Dys=F) & 0.1 & \\
\hline $\mathrm{P}(\mathrm{Ch}=\mathrm{T})$ & 0.92 & $P($ Ch $)=P($ State_Choking) \\
\hline$P(C h=F)$ & 0.08 & \\
\hline $\mathrm{P}(\mathrm{N}=\mathrm{T})$ & 0.86 & $P(N)=P($ Notify $)$ \\
\hline $\mathrm{P}(\mathrm{N}=\mathrm{F})$ & 0.14 & \\
\hline
\end{tabular}

BBN Structure: The methodology of building BBN structure is intuitive, by using the domain-specific ontology and deriving the BBN structure as discussed in the previous sections.

BBN Parameters: After defining the BBN structure (Nodes and arcs between nodes) and the given training set with the prior probabilities. We can now import this training set to Netica depending on the BBN nodes as a learning data set to overcome by the conditional probability of each node.

Below is a table (table 2) showing the conditional probabilities calculated in Netica:

Table 2. Conditional Probability Table Generated by Netica. 


\begin{tabular}{|c|c|c|c|c|}
\hline Node: Notify & \multirow[b]{2}{*}{$\boldsymbol{\nabla}$} & & \multirow{2}{*}{\begin{tabular}{|l} 
Apply \\
Reset
\end{tabular}} & \multirow{2}{*}{\begin{tabular}{|c|} 
OK \\
Close
\end{tabular}} \\
\hline Chance & & & & \\
\hline Activity_Eating & WearingAssist_DysphagiaAssist & StateName_Choking & False & True \\
\hline False & False & False & .75 & .25 \\
\hline False & False & True & .666667 & .333333 \\
\hline False & True & False & .5 & .5 \\
\hline False & True & True & .2 & .8 \\
\hline True & False & False & .5 & .5 \\
\hline True & False & True & .75 & .25 \\
\hline True & True & False & .5 & .5 \\
\hline True & True & True & .0465116 & .953488 \\
\hline
\end{tabular}

\subsection{Results}

The following information are monitored from a sample of PD patients to test PHEN dysphagia assist:

- Concentrating on the most important activity by a percentage of $86.8 \%$

- $88.7 \%$ of the patients wear PHEN dysphagia assist

- $90.6 \%$ the dysphagia

Armed with these statistics we could set up the following Bayes net (Fig. 5):

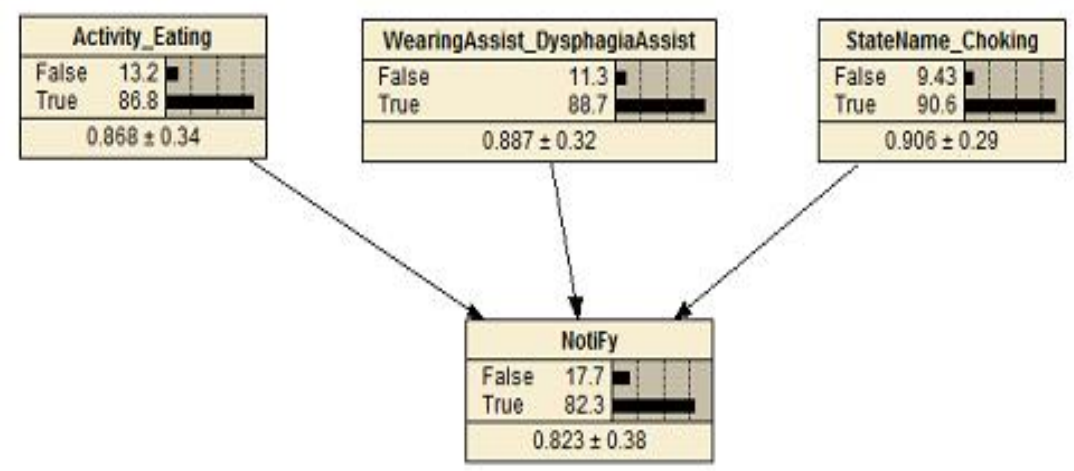

Fig. 5. Netica Software belief network example developed for PHEN model.

\subsection{Inference via $B B N$}

Inferring over the net which gives information about the activity, wearable assistant, state, and alarm notification may answer many useful questions. For instance, "if the alarm gives a notification, what is the probability such that the patient is eating, choking and wearing his wearable assistant? ". Figure 6 shows a sample inference message that given by Netica. 
Given a True Activity_Eating,

the probability of WearingAssist_DysphagiaAssist is 0.954

Given a True Notify,

the probability of Activity Eating and

WearingAssist_DysphagiaAssist is 0.953

Given a True Activity_Eating,

the probability of state_Choking is 0.92

Fig. 6. Inference Message.

Table 3- shows a list of inferences with their values that are computed by Netica and can be used as a base for decision making.

Table 3. List of inferences generated by Netica.

\begin{tabular}{|l|l|}
\hline $\mathrm{P}(\mathrm{Ea}=\mathrm{T} / \mathrm{N}=\mathrm{T})$ & 0.953 \\
\hline $\mathrm{P}(\mathrm{Ea}=\mathrm{T} / \mathrm{N}=\mathrm{F})$ & 0.046 \\
\hline $\mathrm{P}(\mathrm{Ea}=\mathrm{T}, \mathrm{Dys}=\mathrm{T} / \mathrm{N}=\mathrm{T})$ & 0.953 \\
\hline $\mathrm{P}(\mathrm{Ea}=\mathrm{T}, \mathrm{Dys}=\mathrm{T} / \mathrm{N}=\mathrm{F})$ & 0.046 \\
\hline $\mathrm{P}(\mathrm{Dys}=\mathrm{T} / \mathrm{Ea}=\mathrm{T})$ & 0.954 \\
\hline $\mathrm{P}(\mathrm{Dys}=\mathrm{T} / \mathrm{Ea}=\mathrm{F})$ & 0.04545 \\
\hline $\mathrm{P}(\mathrm{Ch}=\mathrm{T}, \mathrm{Dys}=\mathrm{T}, \mathrm{Ea}=\mathrm{T} / \mathrm{N}=\mathrm{T})$ & 0.7754 \\
\hline $\mathrm{P}(\mathrm{Ch}=\mathrm{T}, \mathrm{Dys}=\mathrm{T}, \mathrm{Ea}=\mathrm{T} / \mathrm{N}=\mathrm{F})$ & 0.224 \\
\hline $\mathrm{P}(\mathrm{Ch}=\mathrm{T} / \mathrm{Ea}=\mathrm{T})$ & 0.92 \\
\hline $\mathrm{P}(\mathrm{Ch}=\mathrm{T} / \mathrm{Ea}=\mathrm{F})$ & 0.08 \\
\hline
\end{tabular}

For example if we want to calculate these probabilities manually:

$\mathrm{P}(\mathrm{Ch}=\mathrm{T}, \mathrm{Dys}=\mathrm{T}, \mathrm{Ea}=\mathrm{T} / \mathrm{N}=\mathrm{T})=$

$\mathrm{P}(\mathrm{N}=\mathrm{T} / \mathrm{Ch}=\mathrm{T}, \mathrm{Dys}=\mathrm{T}, \mathrm{Ea}=\mathrm{T}) * \mathrm{P}(\mathrm{Ch}=\mathrm{T}, \mathrm{Dys}=\mathrm{T}, \mathrm{Ea}=\mathrm{T}) / \mathrm{P}(\mathrm{N}=\mathrm{T})=0.7754$

Finally BBN are useful everywhere where modeling an uncertain reality is involved (and hence probabilities are present), and, in the case of decision nets, wherever it is helpful to make intelligent, justifiable, quantifiable decisions that will maximize the chances of a desirable outcome.

By interpreting our results. Inference is used to calculate the probability of any event conditioned by any another event, which helps in handling uncertain contexts by predicting the probability of the unknowns. We can compute the probability of the choking knowing that the patient is not eating (these two nodes are independent). So now our PHEN system can decide when the alarm must notify the patient knowing the state, activity and wearable assistant of the patient. 


\section{Results and Discussion}

This paper proposed PHEN, a real-time system that supports the Parkinson patients in unsupervised environments. The framework is a personalized wearable assistant that 0 detects the dysphagia events and it gives the patient an appropriate audio feedback.

PHEN is a multifunctional system that exceeds the role of being just a daily life assistant, to provide a set of services that varies between healthy, treatment, and exercising. The main point of strength of PHEN is that after a period of time of using the system as an assistive device or as a training device, the patients will experience an overall improvement of their swallowing difficulties.

We exploited the advantages of the ontology to the description of the general context of the patient's domain and captured as many concepts as possible to succeed a complete assistance personalized and fully adapted to the user needs. Indeed, as uncertainty can't be avoided, we used the probabilistic model to handle it by describing the relation chains and the conditional probability dependencies between the concepts. Moreover the Bayesian belief network is constructed and held to take the appropriate decision relative to the patient situation.

Moreover, a use case scenario was used to introduce our approach, which captures all the supported functionality and at the end we presented a demonstration to show a live application of the approach with real conditions and users. Moreover, PHEN system is applied and tested on real data using Netica software and detects dysphagia symptom by $82.3 \%$.

Finally as a future work we would suggest to provide mechanisms at the level of activity recognition to handle and support parallel activities and to confirm the result of the activity recognition, and same for the raw data collected by the sensors. Moreover, we aim to develop our approach to be used in reality to help PD patients.

Acknowledgments. This work is supported by the Department of Computer Science at the University of Quebec at Chicoutimi, Quebec Canada; and by the Faculty of Science at the Lebanese University.

\section{References}

1. Bilal Ahmed, Young-Koo Lee, Sungyoung Lee and Yonil Zhung, "Scenario Based Fault Detection in Context-Aware Ubiquitous Systems using Bayesian Networks". In Proceedings of Computational Intelligence for Modelling, Control and Automation, 2005 and International Conference on Intelligent Agents, Web Technologies and Internet Commerce, International Conference on, Vol(1), pages 414, Vienna, 28-30 Nov. 2005.

2. Michael Angermann, Patrick Robertson, Thomas Strang, "Issues and Requirements for Bayesian Approaches in Context Aware Systems". In Proceedings of Springer Berlin Heidelberg, pages 235, 2005. 
3. Ben-Gal I., Bayesian Networks, In: Ruggeri F., Faltin F. \& Kenett R. (Eds.), Encyclopedia of Statistics in Quality and Reliability, John Wiley \& Sons (2007).

4. M Bächlin , M Plotnik, D Roggen, I Maidan, JM Hausdorff, N Giladi, G Tröster ,Wearable assistant for Parkinson's disease patients with the freezing of gait symptom". In Proceedings of IEEE transactions on information technology in biomedicine: a publication of the IEEE Engineering in Medicine and Biology Society, 2010.

5. M. J. Druzdzel and L. C. Van Der Gaag. "Building bayesian networks: Where do the numbers come from?". In Proceedings of IEEE Transactions on Knowledge and Data Engineering, 12:481-486, 2000 .

6. S.O. Funtowicz and J.R. Ravetz, "Uncertainty and Quality in Science for Policy". Kluwer Academic Publishers, Dordrecht, 1990.

7. Tao Gu, Hung Keng Pung, Da Qing Zhang, “A Bayesian approach for dealing with uncertain contexts". In proceedings of Austrian Computer Society, 2004.

8. National Institutes of Health " What Causes Parkinson's Disease?", Internet: http://nihseniorhealth.gov/, 2014.

9. Hamid Mcheick, Malak Khreis, Mohammad Al-Kalla, and Hala Sweidan, "CARE: Context-Aware Reliable Engine Health for Traffic Monitoring System”. In Proceedings of UKSim-AMSS 16th International Conference on Computer Modelling and Simulation, pages 410, 2014.

10. G. Klir and M. Wierman. Uncertainty-Based Information: Elements of Generalized Information Theory. (2nd edition). [On-line] Available: $\mathrm{http} / / / \mathrm{www}$.amazon.com/Uncertainty-Based-Information-Generalized-Fuzziness Computing/dp/3790812420. [October 19, 1999].

11. R. Lerner, U. Parr. Bayesian fault detection and diagnosis in dynamic systems. In Seventeenth National Conference on Artificial Intelligence, pages 531-537. AAAI, 2000.

12. Sinziana Mazilu, Michael Hardegger, Ulf Blanke, Gerhard Tröster, Eran Gazit, Moran Dorfman and Jeffrey M Hausdorff, " GaitAssist: A Wearable Assistant for Gait Training and Rehabilitation in Parkinson's Disease”. In Proceedings of IEEE Percom (DEMO technical details about the CHI system) [Best Demo Award], 2014.

13. Tam Van Nguyen, Wontaek Lim, Huy Anh Nguyen, Deokjai Choi and Chilwoo Lee, "Context Ontology Implementation for Smart Home". In Proceedings of CoRR abs/1007.1273,2010.

14. Judea Pearl, "Probabilistic Reasoning in Intelligent Systems: Networks of Plausible Inference", Morgan Kaufmann, 1988.

15. Binh An Truong, Young-Koo Lee and Sung-Young Lee, "Modeling and Reasoning about Uncertainty in Context-Aware Systems". In Proceedings of e-Business Engineering. ICEBE 2005. IEEE International Conference on, page: 102, 12-18 Oct. 2005.

16. Binh An Truong, Young-Koo Lee and Sung-Young Lee, "Modeling Uncertainty in Context-Aware Computing". In Proceeding of Computer and Information Science, 2005. Fourth Annual ACIS International Conference on , pages: 676, 2005.

17. Norsys Software Corp., "https://www.norsys.com/netica.html"

18. Hamid Mcheick, Hassan Sbeity, Hussein Hazimeh, Jihad Naim, and Mohamed Alameh. Context Aware Mobile Application Architecture (CAMAA) for Health Care Systems : Standardization and abstraction of context aware layers, 2014 IEEE International Humanitarian Technology Conference (IHTC-IEEE). June 1-4, 2014, Montreal, Canada.

19. Hamid Mcheick. Modeling Context Aware Features for Pervasive Computing, The 5th International Conference on Emerging Ubiquitous Systems and Pervasive Networks (EUSPN-2014), Journal title: Procedia Computer Science, Procedia Computer Science (2014), pp. 135-142, DOI information: 10.1016/j.procs.2014.08.022. Elsevier, September 22-25, Halifax, Canada, 2014. 